\title{
BMJ Open Progress in voluntary medical male circumcision for HIV prevention supported by the US President's Emergency Plan for AIDS Relief through 2017: longitudinal and recent cross-sectional programme data
}

\author{
Stephanie M Davis, ${ }^{1}$ Jonas Z Hines, ${ }^{1}$ Melissa Habel, ${ }^{1}$ Jonathan M Grund, ${ }^{1}$ \\ Renee Ridzon, ${ }^{2}$ Brittney Baack, ${ }^{3}$ Jonathan Davitte, ${ }^{4}$ Anne Thomas, ${ }^{4}$ \\ Valerian Kiggundu, ${ }^{5}$ Naomi Bock, ${ }^{1}$ Paran Pordell, ${ }^{3}$ Caroline Cooney, ${ }^{2}$ Irum Zaidi, ${ }^{2}$ \\ Carlos Toledo ${ }^{1}$
}

To cite: Davis SM, Hines JZ, Habel M, et al. Progress in voluntary medical male circumcision for HIV prevention supported by the US President's Emergency Plan for AIDS Relief through 2017: Iongitudinal and recent cross-sectional programme data. BMJ Open 2018;8:e21835. doi:10.1136/ bmjopen-2018-021835

\section{- Prepublication history for} this paper is available online. To view these files, please visit the journal online (http://dx.doi. org/10.1136/bmjopen-2018021835).

Received 22 January 2018 Revised 26 July 2018

Accepted 30 July 2018
Check for updates

(C) Author(s) (or their employer(s)) 2018. Re-use permitted under CC BY-NC. No commercial re-use. See rights and permissions. Published by BMJ.

For numbered affiliations see end of article.

Correspondence to Dr Stephanie M Davis; vic6@cdc.gov

\section{ABSTRACT}

Objective This article provides an overview and interpretation of the performance of the US President's Emergency Plan for AIDS Relief's (PEPFAR's) male circumcision programme which has supported the majority of voluntary medical male circumcisions (VMMCs) performed for HIV prevention, from its 2007 inception to 2017, and client characteristics in 2017.

Design Longitudinal collection of routine programme data and disaggregations.

Setting 14 countries in sub-Saharan Africa with low baseline male circumcision coverage, high HIV prevalence and PEPFAR-supported VMMC programmes.

Participants Clients of PEPFAR-supported VMMC programmes directed at males aged 10 years and above.

Main outcome measures Numbers of circumcisions performed and disaggregations by age band, result of HIV test offer, procedure technique and follow-up visit attendance.

Results PEPFAR supported a total of 15269720 circumcisions in 14 countries in Southern and Eastern Africa. In 2017, 45\% of clients were under 15 years of age, $8 \%$ had unknown HIV status, $1 \%$ of those tested were HIV+ and $84 \%$ returned for a follow-up visit within 14 days of circumcision.

Conclusions Over 15 million VMMCs have been supported by PEPFAR since 2007. VMMC continues to attract primarily young clients. The non-trivial proportion of clients not testing for HIV is expected, and may be reassuring that testing is not being presented as mandatory for access to circumcision, or in some cases reflect test kit stockouts or recent testing elsewhere. While VMMC is extremely safe, achieving the highest possible follow-up rates for early diagnosis and intervention on complications is crucial, and programmes continue to work to raise follow-up rates. The VMMC programme has achieved rapid scale-up but continues to face challenges, and new approaches may be needed to achieve the new Joint United Nations
Strengths and limitations of this study

The large dataset covers 11 years of programme data on over 15 million men.

- US President's Emergency Plan for AIDS Reliefsupported clients constitute a large majority of all voluntary medical male circumcision clients, making client characteristics reported here likely to be representative of the programme as a whole.

- The multicountry nature of the data allows determination of client characteristics common across settings.

- The routine reporting system is aggregate only, and collected data do not support analyses of associations between client characteristics.

- Data are not complete for all client characteristics (disaggregations) in all countries.

Programme on HIV/AIDS goal of 27 million additional circumcisions through 2020.

\section{INTRODUCTION}

Male circumcision, the removal of the foreskin, reduces a man's risk of acquiring HIV through heterosexual exposure by approximately $60 \%{ }^{1-3}$ Since its adoption as a recommended HIV prevention strategy by the WHO and Joint United Nations Programme on HIV/AIDS (UNAIDS) in 2007, ${ }^{4}$ voluntary medical male circumcision (VMMC) has become a core component of the global HIV prevention portfolio through support by national and global programmes, such as the US President's Emergency Plan for AIDS Relief (PEPFAR) and the Global Fund 
to Fight AIDS, Tuberculosis and Malaria. ${ }^{5}$ The WHO/ UNAIDS guiding document for VMMC from 2012 to 2016 recommended scale-up of VMMC in 14 countries in Southern and Eastern Africa with high HIV prevalence, low circumcision coverage and primarily heterosexual HIV transmission, ${ }^{6}$ with an initial goal of circumcising 20 million men to achieve $80 \%$ coverage among males aged 15-49 years in these countries by 2016. As a one-time biomedical intervention conferring lifelong partial protection from HIV, and the only prevention method specifically targeted at HIV-negative heterosexual men, VMMC complements other behavioural and clinical prevention interventions such as condom promotion, reduction in number of sex partners, pre-exposure prophylaxis and treatment-as-prevention.

VMMC services were launched progressively by ministries of health in each of the recommended 14 countries between 2007 and 2011, primarily with PEPFAR support. PEPFAR-supported VMMCs are surgical or procedural interventions often lasting $5-15 \mathrm{~min},{ }^{7}$ provided by physicians or nurses under local anaesthesia. They are offered with the WHO-recommended minimum package $^{4}$ of accompanying services, including the offer of HIV testing and linkage to care and treatment for HIV-positive individuals, HIV risk-reduction education, condom provision and sexually transmitted infection (STI) screening and treatment or referral. Over the lifetime of the programme, several additional key innovations with potential to impact the volume and client demographics of the programme have also been incorporated. These include the introduction of WHO-prequalified circumcision devices as alternatives to conventional surgery; the introduction of WHO 'Models for Optimizing Volume and Efficiency'; the identification of the age group of 15-29years as the focal demographic for rapidly reducing population-level HIV incidence through VMMC $^{9}$; enhancement of safety measures based on accumulating data, particularly for adolescents based on their unique surgical safety requirements; introduction of policies on tetanus vaccination prior to $\mathrm{VMMC}^{10}$ and increased emphasis on linking clients testing HIV positive to care and treatment, ${ }^{11}$ to support WHO/UNAIDS treatment scale-up goals. ${ }^{12}$

All PEPFAR-supported VMMC programmes report on key programme outcomes, including the total VMMCs performed in each country and select disaggregations for all clients: client age range, result of HIV test offer, circumcision technique (device or surgical) and adherence to routine postoperative follow-up. These indicators provide an overview of the accomplishments and key current attributes of the PEPFAR VMMC programme. This article presents all PEPFAR-supported VMMCs performed since programme inception in 2007, compares key disaggregations from US fiscal year 2017 with those from 2015 and 2016, and discusses future challenges and directions for the programme. The primary objective of this paper is to describe PEPFAR's VMMC programme, and identify in which aspects the programme is performing well and in which gaps and challenges remain and should be prioritised. Making key demographic data on VMMC clients available may also support refining projections around impact. Client characteristics described here are expected to be reasonably representative of the global VMMC clientele to date because PEPFAR supports the majority of VMMCs conducted globally (approximately $82 \%$ through 2017), ${ }^{13}$ and because VMMC donors operate under the common WHO minimum package service structure.

\section{METHODS}

Data reported by PEPFAR-supported VMMC programmes are drawn initially from site-level client registers and records, and reported by the implementing partners at the site level to PEPFAR in each host country. Data are then cleaned in-country; and then reported to PEPFAR's central coordinating body, the Office of the Global AIDS Coordinator. Implementing partners also report to their host country governments, following procedures agreed on in-country; this may occur independently or may use the same data stream. Data are currently reported to PEPFAR on a quarterly basis, and then grouped by US Government fiscal year which runs from 1 October to 30 September, rather than by calendar year. These numerical data are now available online ${ }^{14}$ and have been used here to calculate percentages where relevant. Online data are subject to ongoing refinement if revised information is received from countries. Monitoring trends across years is possible, but prior to 2015, fewer VMMC disaggregations were collected.

The disaggregations reported for VMMCs performed, and definitions where needed, are listed in table 1 . Their rationales and context are summarised below. Not all sites are able to collect and report circumcision data by each disaggregate for all clients. Therefore, percentages presented here for each disaggregate are calculated only among clients for whom data on that disaggregate were reported.

1. Client age range: Age ranges represent those selected for PEPFAR routine reporting to support fine analysis of client age patterns. PEPFAR's prioritisation of, or 'age pivot' to, the age range of 15-29years began in fiscal year 2016. Ages below 15 years constitute the largest combined proportion of VMMCs performed, but will have delayed impact on the HIV epidemic due to the delay until sexual debut and other risk behaviours. These age ranges are collapsed together in this report. 2. Result of HIV test offer at VMMC site: Under PEPFAR, ${ }^{15}$ UNAIDS $^{16}$ and $\mathrm{WHO}^{4}$ guidance, HIV testing at the VMMC site should be encouraged but is not required for access to VMMC, and clinically eligible clients who do test may proceed with VMMC regardless of their results. As a disaggregation within the cohort of clients receiving VMMC, this reporting does not include men who were tested at VMMC sites but then decided against VMMC after receiving a positive HIV test result. This may not be unusual, as men testing positive are 
Table 1 Disaggregations and categories for PEPFARsupported VMMC programme reporting on VMMCs performed, 2016

\begin{tabular}{ll}
\hline Disaggregation & Client categories \\
\hline Age & $<61$ days (early infant male \\
& circumcision). \\
& 2 months to $<10$ years (not funded by \\
& PEPFAR due to safety concerns). \\
& 10-14 years (this and younger \\
& categories are collapsed here as '<15'). \\
& $15-19$ years. \\
& $20-24$ years. \\
& $25-29$ years. \\
& 30-49years. \\
& $50+$ years. \\
& HIV+. \\
Result of HIV & HIV-. \\
test offer at & Unknown: includes clients with \\
VMMC site & undocumented or indeterminate status \\
& and those not tested at the VMMC site \\
& for any reason. \\
& Surgical. \\
Technique & Device-based. \\
Follow-up visit & Returned for at least one postoperative \\
attendance & follow-up visit within 14 days of surgery. \\
& Did not return within 14 days. \\
\hline
\end{tabular}

PEPFAR, US President's Emergency Plan for AIDS Relief; VMMC, voluntary medical male circumcision.

counselled that circumcision will not affect the course of their HIV infection and may choose not to proceed.

3. Circumcision technique used: Circumcision devices offer an alternative to surgery, and advantages include faster procedures and potentially avoiding the injection of anaesthesia and incision of living tissue. Initially, PEPFAR-supported circumcision was exclusively surgical, using one of three WHO-recommended techniques: forceps-guided, dorsal slit and sleeve resection. ${ }^{17}$ With WHO prequalification of the PrePex device in $2013^{18}$ and ShangRing in 2015, ${ }^{19}$ some ministries of health have introduced device-based services as additional options for clients. Both PrePex and ShangRing devices remain on the penis for 7 days and are removed by a provider during a follow-up visit. Anatomical and age-based eligibility criteria vary between devices and can be stricter than surgical eligibility criteria, restricting the pool of eligible clients. ${ }^{20}$ Numbers of circumcisions performed using a device therefore reflect national policies and eligibility for, as well as availability and client uptake of, these alternative methods. They also reflect the impact of tetanus mitigation policies as discussed below.

4. Follow-up visit attendance: PEPFAR-supported programmes follow fixed schedules for routine postoperative follow-up set by national ministries of health to ensure client safety. These often include a return visit at days 2 and 7 after surgery, or day 7 after device placement (for device removal). In 2015 and 2016, the PEPFAR follow-up indicator was only reported among surgical cases which therefore constituted the denominator for determining follow-up percentage; in 2017, it was calculated among all circumcisions. Follow-up was only reported on if it took place, so that clients who did not follow up cannot be distinguished from those whose follow-up status is unknown.

Comparison data on country-specific total annual VMMC volume (including both PEPFAR-supported and non-PEPFAR-supported procedures) are drawn from the most recent WHO VMMC Progress Brief, covering 20072017. ${ }^{13}$ Data on PEPFAR's 2017 country-level VMMC targets are drawn from annual PEPFAR Budget and Target reports. $^{21}$

\section{Patient and public involvement}

Because this manuscript reported programme data rather than research data, no research question or study design was formulated for data collection. Data collected instead consisted of indicators designed to monitor programme achievements. VMMC clients were beneficiaries choosing to uptake a public health interventions, not research participants. However, satisfied VMMC clients were sometimes involved in recruiting peers for VMMC. Results are publicly available online as referenced above.

\section{RESULTS}

From PEPFAR fiscal year 2007 to 2017, a total of 15269720 PEPFAR-supported VMMCs were performed in 14 countries in Southern and Eastern Africa. From 2010 to 2013, annual VMMC numbers approximately doubled annually, reaching 2794808 in 2014. In 2015, 2573273 VMMCs were performed, followed in 2016 by 2290 200; these represent the third and fourth highest annual totals. The year 2017 saw an increase to 3383 444, the highest annual total to date. Over half $(54 \%)$ of PEPFAR-supported circumcisions performed since the programme's inception were performed in 2015-2017 (figure 1 and table 2). In 2017, PEPFAR-supported VMMCs constituted $84 \%$ of all VMMCs reported by WHO, and $65 \%$ or more of reported VMMCs in each individual country except Malawi. ${ }^{12}$ PEPFAR-supported VMMCs conducted in 2017 achieved 92\% (3 383 444/3 666 356) of PEPFAR targets for that year, with nine countries reaching $90 \%$ or more of their targets.

Twelve countries provided $>85 \%$ data completeness across all disaggregations in 2017 for which completeness was calculable. By disaggregate, 3278130 clients had age data $(387736$ missing $=12 \%) ; 3309077$ had result of HIV test offer (including unknown status; 356789 missing=11\%); 3282987 had technique data (382879 missing=12\%); of whom 2736851 had a reported follow-up within 14 days. Because programmes only reported the number of clients who received follow-up in 2017, and did not separately report the number which did not, we are unable to assess completeness for this disaggregate 


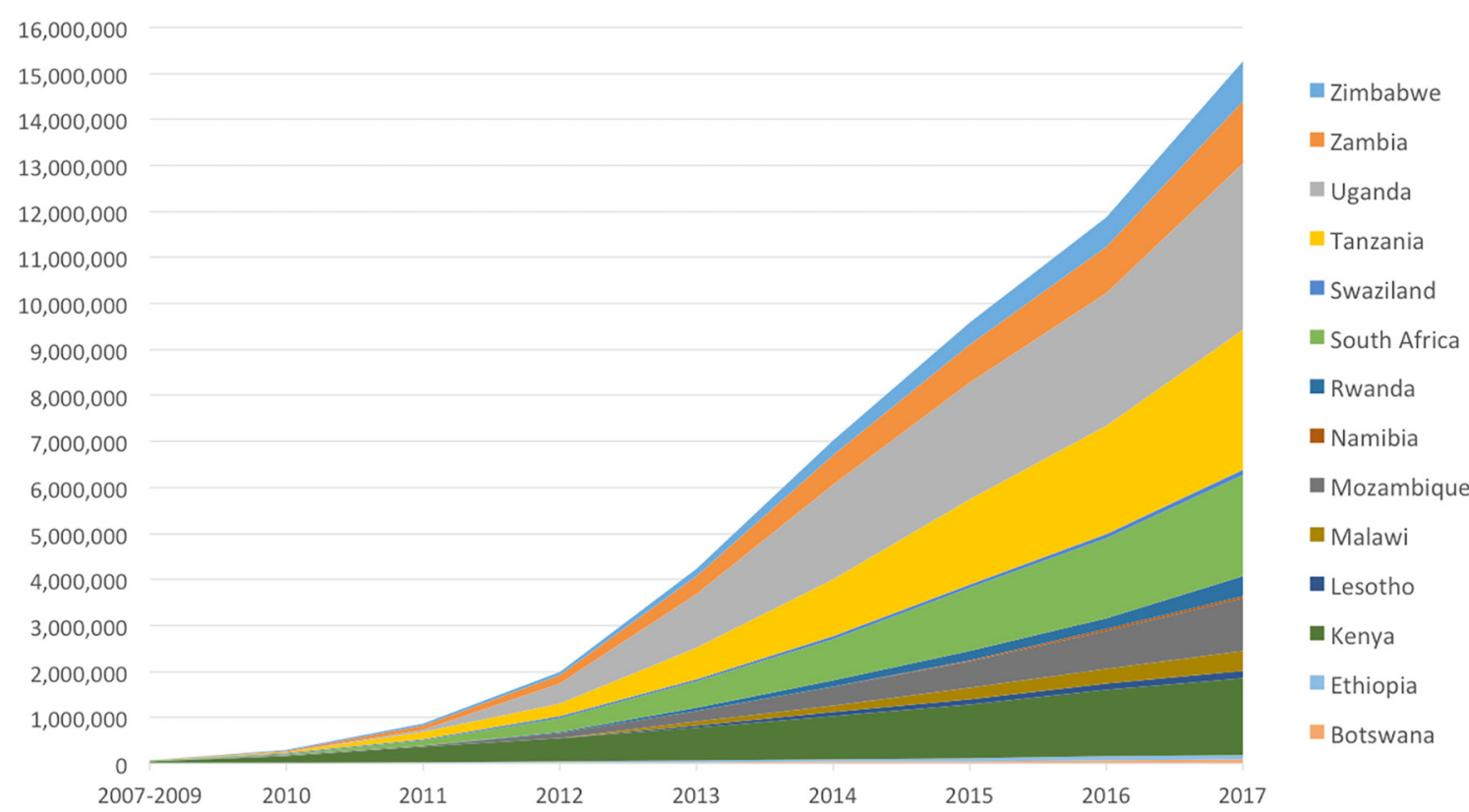

Figure 1 Cumulative number of US President's Emergency Plan for AIDS Relief-supported voluntary medical male circumcisions by country, 2007-2017.

(but see table 2 footnote). Where data completeness was less than the $85 \%$ threshold we selected for this analysis for a given disaggregate (sum of all VMMCs across categories for that disaggregate, divided by total VMMCs performed), values are in bold (table 3 ).

In 2017, 48\% of VMMC clients were within the 15-29 years age range, to which PEPFAR shifted focus that year, a relatively stable proportion from $46 \%$ in 2016 and $48 \%$ in 2015. There was wide variation among countries, ranging from $26 \%$ in Botswana to $69 \%$ in Rwanda. In contrast, $45 \%$ of PEPFAR VMMC clients were within the $<15$ years range, representing a stable increase, along with $46 \%$ in 2016, from $42 \%$ in 2015. Eight countries experienced an increase in the proportion of clients in the $<15$ years age range from 2016 to 2017. This shift was largest in Rwanda $(5 \%-28 \%)$, Botswana $(45 \%-66 \%)$ and Namibia $(12 \%-$ $34 \%$ ), all relatively small programmes. However, other programmes, like South Africa, did successfully achieve the reverse ( $43 \%$ in 2017 , from $51 \%$ in 2016). Countries which had previously shifted in 2016 towards younger clientele included several large programmes which also showed decreases in overall achievements in that year, such as Tanzania, South Africa and Zambia, though in the first two cases this trend reversed in 2017.

Overall, $8 \%$ of clients had 'unknown' HIV status in 2017, as compared with $9 \%$ in 2016 and $7 \%$ in 2015, with individual country values ranging from $0 \%$ in Rwanda and Zimbabwe to $50 \%$ in Lesotho; other countries with high 'unknown' rates were Ethiopia at 32\% and Namibia at $44 \%$. Among clients tested for HIV, $1 \%$ were positive, essentially unchanged from 2015 through 2017 , with individual country positivity varying between $0 \%$ in multiple countries and 3\% in eSwatini (formerly Swaziland).
Device-based circumcision techniques constituted a small percentage of circumcisions in all countries in 2017, accounting for $3 \%$ of VMMCs across countries (down from 5\% in 2015 and 4\% in 2016), ranging between $0 \%$ in many countries and 53\% in Rwanda. Botswana and Zimbabwe showed substantial respective drops from $28 \%$ to $0 \%$ and $27 \%$ to $5 \%$.

Postprocedure follow-up rates among all clients were $84 \%$ overall (excluding Zimbabwe), higher than the $72 \%$ in 2015 and $78 \%$ in 2016. These rates ranged between $59 \%$ in South Africa and $100 \%$ in Rwanda, falling at or above $75 \%$ in 11 countries.

\section{DISCUSSION}

VMMC has undergone a scale-up of historic proportions within global health, comparable with the scale-up of HIV treatment. ${ }^{22}$ This manuscript demonstrates that overall programme volume is high and increasing, but there is progress to be made in increasing volume further to pursue Fast Track targets, attracting older clients with higher HIV risk and, in some places, raising postoperative follow-up rates to ensure complications are managed promptly.

With respect to programme volume, over 15 million PEPFAR-supported VMMCs were performed between 2007 when support began and 2017, making VMMC a major prevention intervention for achieving the PEPFAR goal of an AIDS-free generation through epidemic control in sub-Saharan Africa. ${ }^{23}$ PEPFAR had committed in 2015 to supporting at least 13 million VMMCs through 2017, ${ }^{24}$ and exceeded that target. Models estimate that global VMMCs conducted through 2016 alone will avert 500000 infections by the end of $2030 .{ }^{12}$ Scale-up has been enabled 


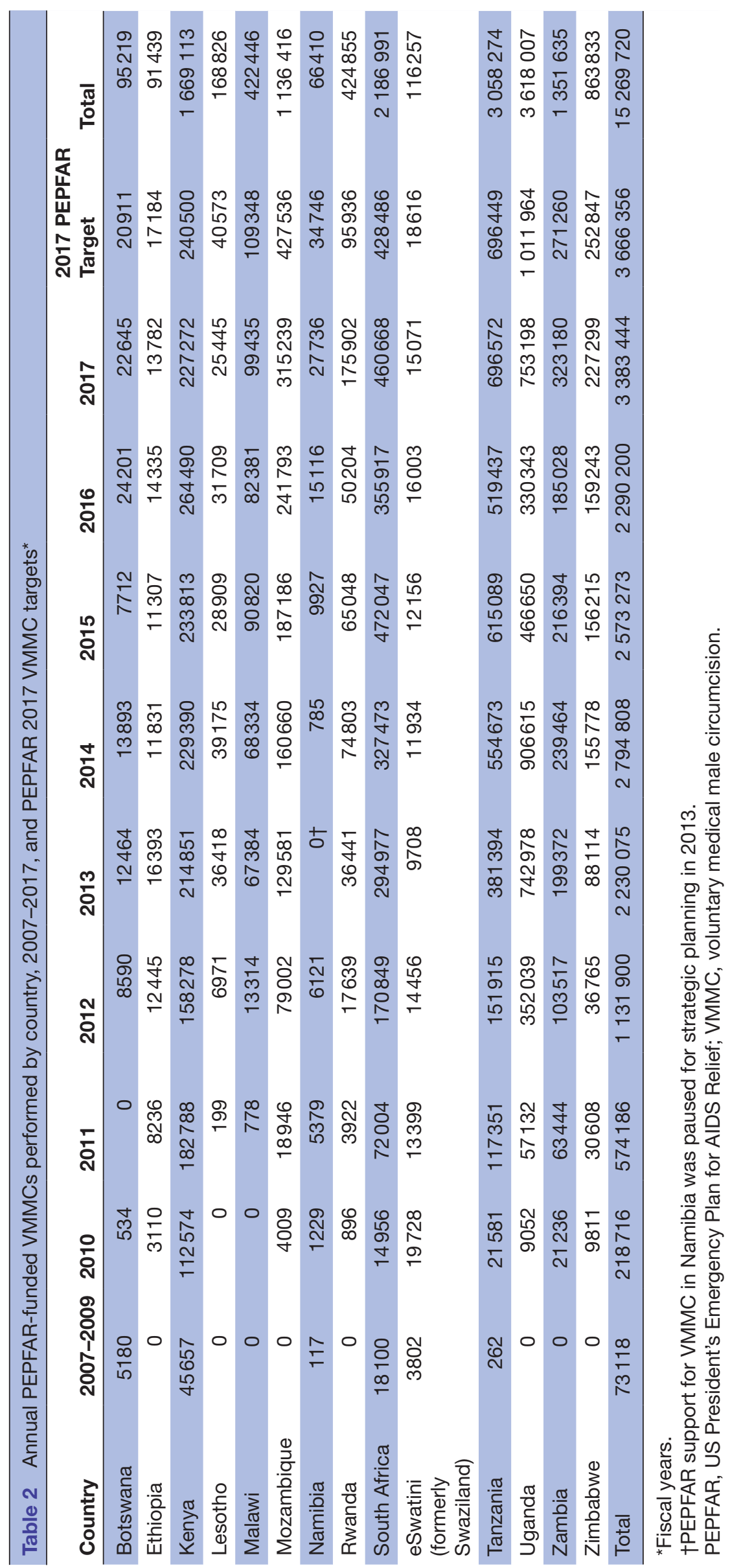


Table 3 Numbers and characteristics of PEPFAR-funded voluntary medical male circumcisions, fiscal year 2017 (1 October 2016-30 September 2017) by country and 2015-2016 totals

\begin{tabular}{|c|c|c|c|c|c|c|c|c|c|c|c|}
\hline \multirow[b]{2}{*}{ Country } & \multirow[b]{2}{*}{ VMMCs } & \multicolumn{6}{|c|}{ Client age range in years $(\%)^{*}$} & \multicolumn{2}{|c|}{$\begin{array}{l}\text { Result of HIV test offer } \\
\text { at VMMC site (\%) }\end{array}$} & \multirow{2}{*}{$\begin{array}{l}\text { Technique: } \\
\text { device } \\
\text { method used } \\
\text { (\%) } \\
\text { (vs surgical) }\end{array}$} & \multirow{2}{*}{$\begin{array}{l}\text { Follow- } \\
\text { up visit } \\
\text { attendance } \\
\dagger(\%)\end{array}$} \\
\hline & & $<15$ & $15-19$ & $20-24$ & 25-29 & $30-49$ & $50+$ & $\begin{array}{l}\text { tested } \\
\text { clients }\end{array}$ & $\begin{array}{l}\text { Unknown } \\
\text { status* }^{*}\end{array}$ & & \\
\hline Botswana & 22645 & 66 & 10 & 9 & 7 & 7 & 1 & 0 & 18 & 0 & 72 \\
\hline Ethiopia & 13782 & 47 & 26 & 14 & 6 & 7 & 0 & 0 & 32 & 0 & 96 \\
\hline Lesotho & 25445 & 56 & 18 & 8 & 6 & 10 & 1 & 1 & 50 & 0 & 90 \\
\hline Malawi & 99435 & 42 & 28 & 15 & 8 & 6 & 0 & 1 & 0 & 0 & 89 \\
\hline Mozambique & 315239 & 45 & 30 & 13 & 6 & 5 & 1 & 2 & 6 & 0 & 81 \\
\hline Namibia & 27736 & 34 & 21 & 15 & 13 & 16 & 1 & 0 & 44 & 0 & 99 \\
\hline Rwanda & 175902 & 28 & 48 & 15 & 6 & 4 & 0 & 0 & 0 & 53 & 100 \\
\hline Tanzania & 696572 & 46 & 26 & 15 & 6 & 6 & 1 & 0 & 13 & 0 & 92 \\
\hline Uganda & 753198 & 46 & 26 & 13 & 8 & 6 & 1 & 1 & 3 & 0 & 86 \\
\hline Zambia & 323180 & 38 & 27 & 16 & 10 & 9 & 0 & 0 & 4 & 0 & 94 \\
\hline Zimbabwe & 227299 & 47 & 25 & 13 & 8 & 7 & 0 & 0 & 0 & 5 & $\ddagger$ \\
\hline Total in 2017 & 3383444 & 45 & 27 & 13 & 8 & 7 & 1 & 1 & 8 & 3 & 84 \\
\hline Total in 2016 & 2290141 & 46 & 27 & 12 & 7 & 7 & 1 & 1 & 9 & 4 & 78 \\
\hline Total in 2015 & 2573238 & 42 & 28 & 13 & 7 & 8 & 1 & 1 & 7 & 5 & 72 \\
\hline
\end{tabular}

Entries in bold indicate $<85 \%$ data completeness.

*Includes clients with undocumented or indeterminate status and those not tested at the VMMC site for any reason.

†Per cent of clients who returned for at least one postoperative follow-up visit within 14 days of surgery or device placement. ҒFollow-up rate in Zimbabwe unknown for 2017. Facilities representing $46 \%$ of all Zimbabwe circumcisions did not report follow-up data, due to delays in adoption of the new follow-up reporting method.

PEPFAR, US President's Emergency Plan for AIDS Relief; VMMC, voluntary medical male circumcision.

not only by dedicated resources, totalling over $\$ 1.5$ billion through 2017 from PEPFAR alone, but also through leadership from ministries of health, rapid expansion of surgical skills and responsibilities to non-physician health cadres, and public outreach campaigns. It has provided millions of men with lifelong partial protection from HIV and some STIs, and connected them with additional HIV and other health services through testing, STI screening and referrals for other health conditions. Infections averted in men through VMMC also help prevent new HIV infections among women and girls, ${ }^{25-27}$ and male circumcision is also associated with lower prevalence of a range of STIs in women. ${ }^{24}$

In light of the high proportion of global VMMCs supported, PEPFAR client data are also expected to provide a reasonably representative view of the global VMMC clientele to date. References to achievements and characteristics below refer to the PEPFAR programme except where otherwise stated.

All VMMC implementing countries continue to scale up VMMC. However, in 2015 and 2016, there were substantial declines in PEPFAR VMMC achievements in Uganda (the largest contributor to VMMC results); these were mirrored by declines in total VMMCs achieved in Uganda. ${ }^{12}$ Service delivery and policy were impacted by the identification of tetanus as a risk among VMMC clients in the Ugandan setting, due to limited historical tetanus immunisation coverage. ${ }^{28-30}$ WHO issues global recommendations for tetanus vaccination to be completed prior to performing circumcisions using device-based methods, ${ }^{31}$ and the Uganda National VMMC Program ultimately required an additional visit for tetanus vaccination prior to VMMC. Similar policies may have also impacted performance in other countries with significant device contributions in prior years (not shown) like Botswana and Rwanda. Additionally, a PEPFAR policy issued in late 2014 discontinued the use of the forceps-guided surgical technique in young adolescents to avoid the associated risk of injury to the immature glans, causing some country programmes to decrease VMMC provision to this age range until they could retrain their VMMC workforces in a more appropriate method. In 2016, Tanzania, South Africa and Zambia also experienced declines (Uganda had not previously reported age data), though these were accompanied by increases in client proportions in the 10-14 age group, and were followed in 2017 by increases in 
overall performance and shifts towards older clientele. These 2016 declines may thus be due to strategic shifts in programme geography and funding. Total programme performance then increased substantially in 2017, with nine countries showing increases over 2016, including these largest programmes. We attribute this recovery to resolution of the challenges described above, combined with increases in available resources.

Country-level PEPFAR VMMC targets are set each year for the following year, based on previous years' achievements, available resources, overall ministry of health targets and remaining unmet need to achieve saturation. In 2017, overall performance was well matched to targets $(92 \%)$, with variations including some countries with marked overperformance (Rwanda and Zambia) and others with high absolute performance but even more ambitious targets (eg, Uganda, with the highest national PEPFAR VMMC performance ever reported). In addition to the policy factors discussed above, reasons for variation in performance against targets can include implementing partner capacity and ambition (partners may choose to surpass their targets once achieved, if funding remains) and unexpected external factors such as civil unrest and healthcare strikes.

VMMC client populations in 2017 were young, with males in the 10-14 years age range representing $45 \%$ of all PEPFAR VMMCs (virtually all VMMCs in clients $<15$ years are in the 10-14years range), as compared with $12 \%$ of the combined male general population of the 14 implementing countries. ${ }^{32}$ This represents a change from the programme's historical client age distribution; from 2010 to 2013 , clients in the $10-14$ years age range characterised no more than one-third of all clients in any year. ${ }^{33}$ The 2015 distribution (42\% of clients were aged $<15$ years) served as the baseline prior to implementation of the 'age pivot'; yet the actual age balance shifted further towards the younger range in $2016(46 \%)$, with only slight recovery in 2017, despite this focus. In some countries, the shift towards younger ages may partially reflect the maturation of the VMMC programme: 'exhaustion' of older age ranges and replacement with younger males. Additional potential barriers which may apply disproportionately to older males include reluctance to abstain from sex for the 6-week healing period, perception of low risk due to having established partners, the opportunity cost of lost wages during recovery and fear of creating perceptions in a stable partner that they intend to seek other sexual partners. ${ }^{34} 35$ Age range of 30 years and above represented a small (8\% in 2017) percentage of VMMC clientele, despite being a sexually active group at risk for HIV, possibly for similar reasons. However, some countries and individual partners did successfully make modest shifts towards older clientele, and identification and broader adoption of their best practices are called for.

VMMC clients also have low HIV positivity compared with the general population, $1 \%$ among all clients in 2015, 2016 and 2017, as compared with 5.5\% (95\% CI
$4.9 \%$ to $6.0 \%$ ) prevalence among males aged $15-49$ years in the Southern and Eastern Africa regions in 2016. ${ }^{36}$ The young age of the client population is almost certainly the major underlying factor; preadolescents and early adolescents have less exposure to sex and consequently less HIV risk and lower HIV prevalence than older age groups. Because HIV test results are not disaggregated by age group, this effect cannot be controlled for in PEPFAR VMMC reporting data. Lower HIV prevalence among VMMC clients could also reflect self-selection of men with health-protecting behaviours into the pool of VMMC clients and/or the effects of messaging promoting VMMC for HIV prevention, leading to the self-exclusion of men who know they are HIV positive (including any who learn they are HIV positive at the VMMC site and consequently opt out of VMMC). Despite this low prevalence, it should be noted that men in sub-Saharan Africa have lower HIV testing uptake than women, ${ }^{37}$ and the VMMC programme provides an opportunity to reach men who might not otherwise undergo testing. The substantial proportion of clients with 'unknown' test results is expected and may reflect the voluntary nature of HIV testing in VMMC, as well as the frequency of HIV test kit stockouts (eg, Ethiopia and Namibia experienced intermittent stockouts in 2017) and in some cases national testing policies (eg, requirements for separate parental consent to test young adolescents).

The fact that few circumcisions were performed by device methods throughout 2015-2017 reflects in part the relatively short period that device-based circumcision has been available, as well as the rigorous country-level vetting process which new devices undergo when being introduced in a new country, ${ }^{38}$ prior to nationwide roll-out. It may also reflect current preferences of clients and programmes. As of publication, all countries implementing PEPFAR-supported VMMC except Ethiopia and Namibia have begun the evaluation process for at least one device, although scale-up of device-based service delivery can take time even after evaluation is completed and policy is in place. Although devices offer several advantages over surgical circumcision, they require more intense post-market safety monitoring tailored to their mechanisms of action, until more VMMCs have been done using these methods. Most recently, uptake of the most commonly used device has also been negatively impacted by the WHO advice and PEPFAR policy related to tetanus vaccination prior to device placement, ${ }^{39}$ driving the decline noted from 2015 to 2017.

Although the follow-up rate of $84 \%$ in 2017 is not precisely comparable with rates in 2015 and 2016 because of the inclusion of device follow-up, the low proportion of the programme represented by device male circumcisions makes the apparent increase likely to constitute a true trend. Follow-up is also likely to be underestimated because all clients without a reported follow-up visit have been treated in calculations as not following up, rather than missing data. This is encouraging, but more remains to be done in countries with low follow-up rates. 
There is evidence that adverse events (AEs) are actually more common among males who do not return for follow-up than among those who do. ${ }^{40}$ While VMMC is extremely safe, ${ }^{41}$ achieving high follow-up rates to allow early $\mathrm{AE}$ diagnosis and intervention is crucial for programme safety.

The findings in this paper are subject to several limitations. These data reflect results supported by PEPFAR, rather than country totals. Additionally, cross-tabulations between disaggregations are not reported, and therefore, it is not possible to determine associations between age range, result of HIV test offer and procedure technique, or control for measured potential confounders. For reasons discussed above, VMMC clients are not representative of the general male population of their countries; they are younger, less likely to be HIV-positive and possibly at lower behavioural risk; findings here are not intended to be generalised outside the client population. Data entry errors such as those discussed above for follow-up, and variations in reporting practice, are possible. Category definitions combine some disparate groups (eg, 'unknown' test results include men who were not tested at VMMC sites for any reason, whether preference, test kit shortage or documentation of recent outside testing.) Finally, though we believe client characteristics are representative of those of all VMMC clients, PEPFAR's focus on the areas of each country with highest absolute HIV burden ${ }^{42}$ could mean that clients of other VMMC programmes in other areas (which may have lower HIV incidence and prevalence, or simply be less densely populated) differ demographically, in unknown ways.

The global VMMC programme's achievements to date have demonstrated the feasibility of rapid scale-up of circumcision coverage, but programme strategy will need to continue evolving for several reasons. Though current achievements have totalled the majority of the initial global target, this target was recently revised under the new UNAIDS Fast Track framework to include 27 million additional circumcisions by the end of $2020,{ }^{43}$ a necessary component of achieving the Fast Track strategy's projected impact. Among these, attracting the PEPFAR priority subpopulation of clients in the 15-29 years age range, and ideally other high-risk clients who will benefit most from VMMC, may require new demand creation approaches and service delivery models. Finally, the updated 2016 WHO/UNAIDS framework for VMMC $^{44}$ calls for circumcision programmes to shift from largely stand-alone service delivery towards greater integration within a broad platform of adolescent and adult male reproductive health. Strategies that reached 'early adopters' may differ from those needed to reach the remaining uncircumcised men, and to do so within this integrated platform. To achieve the ambitious goals in this framework, substantial engagement and increased resource commitments from stakeholders other than PEPFAR, including national ministries of health, will be needed.

\section{Author affiliations}

${ }^{1}$ Division of Global HIV and TB, HIV Prevention Branch, Voluntary Medical Male Circumcision Team, US Centers for Disease Control and Prevention, Atlanta, Georgia, USA

${ }^{2}$ President's Emergency Plan for AIDS Relief, Office of the U.S. Global AIDS Coordinator and Health Diplomacy, Washington, District of Columbia, USA ${ }^{3}$ Division of Global HIV and TB, Monitoring, Evaluation, and Data Analytics Branch, Clinical Monitoring and Evaluation Team, US Centers for Disease Control and Prevention, Atlanta, Georgia

${ }^{4}$ US Department of Defense HIV/AIDS Prevention Program (DHAPP), Naval Health Research Center, San Diego, California, USA

${ }^{5}$ United States Agency for International Development, Global Health Bureau, Office of HIV/AIDS, Prevention, Care and Treatment Division, Washington, District of Columbia, USA

Contributors SMD drafted and finalised the manuscript. JZH, MH, JMG, RR, JD, AT, VK, CC and NB revised the manuscript.BB and PP revised the data and manuscript. CT conceived of the paper, obtained the data and revised the manuscript. IZ oversaw all data collection.

Funding It was supported in part by the President's Emergency Plan for AIDS Relief (PEPFAR) through the Centers for Disease Control and Prevention (CDC).

Competing interests None declared.

Patient consent Not required.

Provenance and peer review Not commissioned; externally peer reviewed.

Data sharing statement As described in this article, the dataset underlying the tables and results is publicly available at https://data.pepfar.net/quarterlyData/.

Open access This is an open access article distributed in accordance with the Creative Commons Attribution Non Commercial (CC BY-NC 4.0) license, which permits others to distribute, remix, adapt, build upon this work non-commercially, and license their derivative works on different terms, provided the original work is properly cited, appropriate credit is given, any changes made indicated, and the use is non-commercial. See: http:// creativecommons.org/licenses/by-nc/4.0/.

\section{REFERENCES}

1. Auvert B, Taljaard D, Lagarde E, et al. Randomized, controlled intervention trial of male circumcision for reduction of HIV infection risk: the ANRS 1265 Trial. PLoS Med 2005;2:e298.

2. Gray RH, Kigozi G, Serwadda D, et al. Male circumcision for HIV prevention in men in Rakai, Uganda: a randomised trial. Lancet 2007;369:657-66.

3. Bailey RC, Moses S, Parker CB, et al. Male circumcision for HIV prevention in young men in Kisumu, Kenya: a randomised controlled trial. Lancet 2007;369:643-56.

4. WHO/UNAIDS Technical Consultation on Male Circumcision and HIV Prevention: Research Implications for Policy and Programming. New Data on Male Circumcision and HIV Prevention: Policy and Programme Implications. Montreux, 6 - 8 March 2007. 2007. http://www.unaids.org/sites/default/files/media_asset/mc_ recommendations_en_1.pdf (accessed 3 Apr 2018).

5. Reed JB, Njeuhmeli E, Thomas AG, et al. Voluntary medical male circumcision: an HIV prevention priority for PEPFAR. J Acquir Immune Defic Syndr 2012;60(Suppl 3):S88-S95.

6. Joint United Nations Programme on HIV/AIDS (UNAIDS). Joint Strategic Action Framework to Accelerate the Scale-Up of Voluntary Medical Male Circumcision for HIV Prevention in Eastern and Southern Africa 2012-2016. 2011. http://www.unaids.org/ sites/default/files/en/media/unaids/contentassets/documents/ unaidspublication/2011/JC2251_Action_Framework_circumcision en.pdf (accessed 3 Apr 2018).

7. Tshimanga M, Mangwiro T, Mugurungi O, et al. A Phase II Randomized Controlled Trial Comparing Safety, Procedure Time, and Cost of the PrePex ${ }^{\mathrm{TM}}$ Device to Forceps Guided Surgical Circumcision in Zimbabwe. PLoS One 2016;11:e0156220.

8. World Health Organization. Considerations for implementing models for optimizing the volume and efficiency of male circumcision services. Geneva: World Health Organization, 2010.

9. Kripke K, Vazzano A, Kirungi W, et al. Modeling the Impact of Uganda's Safe Male Circumcision Program: Implications for Age and Regional Targeting. PLoS One 2016;11:e0158693. 
10. Tetanus and voluntary medical male circumcision: risk according to circumcision method and risk mitigation. Report of the WHO Technical Advisory Group. 2016 (accessed 3 Apr 2018).

11. PEPFAR. The President's Emergency Plan for AIDS Relief Technical Considerations 2015: Provided by PEPFAR Technical Working Groups for 2015 Country Operational Plans and Regional Operational Plans. 2015. http://www.pepfar.gov/documents/organization/237687. pdf (accessed 3 Apr 2018).

12. Joint United Nations Programme on HIV/AIDS (UNAIDS). 90-90-90: An ambitious treatment target to help end the AIDS epidemic. 2014. http://www.unaids.org/sites/default/files/media_asset/90-90-90_en_ $0 . p d f$

13. World Health Organization. WHO progress brief on voluntary medical male circumcision (VMMC) for HIV prevention in 14 priority countries in eastern and southern Africa, July 2018. 2018. https://www. malecirc umcision.org/resourc e/who-progress-brief -voluntarymedical-m ale-circumcision-hiv -prevention-july-2018 (accessed 15 Aug 2018).

14. PEPFAR. Quarterly Results. https://data.pepfar.net/quarterlyData/ (April 3, 2018).

15. PEPFAR's Best Practices for Voluntary Medical Male Circumcision Site Operations: A service guide for site operations. http://www. malecircumcision.org/resources/documents/VMMC\%20Best\% 20Practices03.04.2013_web.pdf (accessed 3 Apr 2018).

16. UNAIDS. Safe, voluntary, informed male circumcision and comprehensive HIV Prevention: programming guidance for decisionmakers on human rights, ethical and legal considerations. 2008. http://www.unaids.org/sites/default/files/media_asset/jc1552_ circumcision_en_0.pdf (accessed 3 Apr 2018).

17. World Health Organization. Manual for Male Circumcision Under Local Anesthesia. 2009. http://www.who.int/hiv/pub/ malecircumcision/who_mc_local_anaesthesia.pdf (accessed 3 Apr 2018).

18. World Health Organization. PrePex device for adult male circumcision for HIV prevention: Information update. 2013. http://www.who.int/hiv/ topics/malecircumcision/prepex_device_update/en/ (accessed 3 Apr 2018).

19. World Health Organization. Information on ShangRing ${ }^{\mathrm{TM}}$ (Generation II, self-locking model) device for voluntary medical male circumcision for HIV prevention. 2015. http://www.who.int/diagnostics_laboratory/ evaluations/150611_shangring_information_notice_v4.pdf?ua=1 (accessed 3 Apr 2018).

20. Tshimanga M, Hatzold K, Mugurungi O, et al. Safety Profile of PrePex Male Circumcision Device and Client Satisfaction With Adolescent Males Aged 13-17 Years in Zimbabwe. J Acquir Immune Defic Syndr 2016;72(Suppl 1):S36-42.

21. PEPFAR FY16 Budget and Target Reports. https://www.pepfar.gov/ countries/cop/c71523.htm (April 26, 2018).

22. UNAIDS. Global AIDS Update 2016. http://www.unaids.org/ sites/default/files/media_asset/global-AIDS-update-2016_en.pdf (accessed 3 Apr 2018).

23. PEPFAR. Strategy for Accelerating HIV/AIDS Epidemic Control (2017-2020). https://www.pepfar.gov/documents/organization/ 274400.pdf

24. U.S President's Emergency Plan for AIDS Relief. Fact Sheet: 2015 United Nations General Assembly Sustainable Development Summit: New PEPFAR HIV Prevention and Treatment Targets. http://www. pepfar.gov/documents/organization/247548.pdf (accessed $3 \mathrm{Apr}$ 2018)

25. Auvert B, Lissouba P, Taljaard D, et al. Male Circumcision: Association With HIV Prevalence, Knowledge, and Attitudes Among Women. 21st Conference on Retroviruses and Opportunistic Infections; 3-6 March 2014; Boston (United States). Topics in Antiviral Medicine 2014;22:504-5.

26. Davis S, Toledo C, Lewis L, et al. Association between HIV and sexually transmitted infections and partner circumcision among women in uMgungundlovu District, South Africa: a cross-sectional analysis of HIPSS baseline data. Abstract TUAC0404. 9th IAS Conference on HIV Science. 2017. http://programme.ias2017.org/ Abstract/Abstract/2833
27. Grund JM, Bryant TS, Jackson I, et al. Association between male circumcision and women's biomedical health outcomes: a systematic review. Lancet Glob Health 2017;5:e1113-e1122.

28. Uganda Ministry of Health. Memo Re: The need for tetanus vaccination for men undergoing circumcision and the continued scale-up of safe male circumcision using PrePex. 2015.

29. Wambi M. "Tetanus Affects Uganda's Medical Male Circumcision Rollout”. Uganda Radio Network. 2016. http://ugandaradionetwork. com/story/tetanus-affects-ugandas-medical-male-circumcision-rollout (accessed 3 Apr 2018).

30. Unicef. Uganda: WHO and UNICEF estimates of immunization coverage: 2015 revision. http://www.who.int/immunization/ monitoring_surveillance/data/uga.pdf (accessed 3 Apr 2018).

31. World Health Organization Technical Advisory Group on Innovations in Medical Male Circumcision. WHO Informal consultation on tetanus and voluntary medical male circumcision, 3 June 2016 - Technical consultation update to the WHO March 2015 meeting report. http:// www.who.int/hiv/pub/malecircumcision/male-circumcision-2016update/en/index1.html (accessed 7 July 2016).

32. "Mid-year Population by Single Year Age Groups - Custom Region". International Programs: United States Census Bureau. 2016. http:// www.census.gov/population/international/data/idb/region.php? $\mathrm{N}=\%$ 20Results $\% 20 \& T=15 \& A=$ aggregate $\& R T=0 \& Y=2016 \& R=-1 \& C=B C, E T$ KE,LT,MI,MZ,WA,RW,SF,WZ,TZ,UG,ZA,ZI

33. Office of the Global AIDS Coordinator. Aggregated PEPFAR internal data, Annual Program Results, 2010-2013. 2014.

34. Hatzold K, Mavhu W, Jasi P, et al. Barriers and motivators to voluntary medical male circumcision uptake among different age groups of men in Zimbabwe: results from a mixed methods study. PLoS One 2014:9:e85051.

35. Nevin PE, Pfeiffer J, Kibira SP, et al. Perceptions of HIV and Safe Male Circumcision in High HIV Prevalence Fishing Communities on Lake Victoria, Uganda. PLoS One 2015;10:e0145543.

36. Regional data: HIV prevalence: Male Adults (15-49). http://aidsinfo. unaids.org/

37. World Health Organization. Factsheet to the WHO Consolidated Guidelines on HIV testing services. 2015 http://www.who.int/hiv/ topics/vct/fact sheet/en/ (accessed 3 Apr 2018).

38. WHO. Framework for clinical evaluation of devices for male circumcision. Geneva 27, Switzerland: WHO Press, 2012. http:// apps.who.int/iris/bitstream/10665/75954/1/9789241504355_eng.pdf. (accessed 3 Apr 2018).

39. World Health Organization. Tetanus and voluntary medical male circumcision: risk according to circumcision method and risk mitigation: Report of the WHO Technical Advisory Group. 12 August 2016. http://apps.who.int/iris/bitstream/10665/250146/1/WHO-HIV2016.19-eng.pdf?ua $=1$ (accessed 3 Apr 2018).

40. Reed JB, Grund J, Liu Y, et al. Implementation and Operational Research: Evaluation of Loss-to-Follow-up and Postoperative Adverse Events in a Voluntary Medical Male Circumcision Program in Nyanza Province, Kenya. J Acquir Immune Defic Syndr 2015;69:e13-23.

41. Cooney C, Toledo C, Kiggundu V, et al. Consolidated overview of notifiable adverse events in the PEPFAR Voluntary medical male circumcision program (January 2015-March 2017). 19th International Conference on HIV and STIs in Africa; Dec 4-9 2017; Abidjan (Cote d'Ivoire).

42. PEPFAR. Fiscal Year 2016 Country/Regional Operational Plan (COP/ ROP) Guidance. https://www.pepfar.gov/reports/guidance/250167. $\mathrm{htm}$ (accessed 5 Apr 2018).

43. UNAIDS. On the Fast Track to End AIDS by 2030: Focus on Location and Population. 2015. http://www.unaids.org/sites/default/files/ media_asset/WAD2015_report_en_part01.pdf (accessed 3 Apr 2018).

44. UNAIDS, World Health Organization. A framework for voluntary medical male circumcision: Effective HIV prevention and a gateway to improved adolescent boys' \& men's health in eastern and southern Africa by 2021 - policy brief. 2016. http://www.who.int/hiv/pub/ malecircumcision/vmmc-policy-2016/en/ 